\title{
Using Interpersonal Process Recall to understand empowerment processes in a collaborative care intervention for people with a
}

diagnosis of psychosis

Elina Baker*1, Ruth Gwernan-Jones ${ }^{1}$, Nicky Britten ${ }^{1}$, Cathy McCabe ${ }^{2}$, Laura Gill ${ }^{2}$, Richard Byng ${ }^{2}$ and Linda Gask ${ }^{3}$

${ }^{1}$ Institute of Health Research, College of Medicine and Health, University of Exeter, Exeter, UK

${ }^{2}$ Community and Primary Care Research Group, Faculty of Medicine and Dentistry, University of Plymouth, Plymouth, UK

${ }^{3}$ Centre for Primary Care, University of Manchester, Manchester, UK

* University of Exeter Medical School, South Cloisters, St Luke's Campus, Exeter, EX1 2LU, UK (e-mail: e.r.baker@ exeter.ac.uk).

Word Count: 6237

This report is independent research funded by the National Institute for Health Research (Programme Grants for Applied Research, PARTNERS2: Development and pilot trial of primary care based collaborative care for people with serious mental illness, RP-PG0611-20004).

Nicky Britten and Richard Byng were partially supported by the National Institute for Health Research (NIHR) Collaboration for Leadership in Applied Health Research and Care South West Peninsula (NIHR CLAHRC South West Peninsula). The views expressed are those of the author(s) and not necessarily those of the NHS, the NIHR or the Department of Health and Social Care.

\section{Data avilability}

The data that support the findings of this study are available on request from the corresponding author [EB]. The data are not publicly available due to containing information that could compromise the privacy of research participants. 


\title{
Using Interpersonal Process Recall to understand empowerment processes in a collaborative care intervention for people with a diagnosis of psychosis
}

\author{
Evaluation of recovery-focused interventions for people with psychosis may be \\ enhanced by the use of Interpersonal Process Recall (IPR). The aim of this study \\ was to examine whether the inclusion of IPR alongside semi-structured \\ interviews in the formative evaluation of a novel collaborative care intervention \\ increased understanding about both practitioner and service user experiences. It \\ also explored the impact of the IPR process on participants. Four service users \\ and the practitioner delivering the intervention participated in semi-structured \\ interviews and an adapted IPR process. The themes identified from different data \\ sources were systematically compared using framework analysis. Semi-structured \\ interviews indicated that the intervention was operating as intended but IPR data \\ revealed exceptions. Practitioner IPR interviews identified practitioner barriers to \\ delivery and fostered critical self-reflection. Service user IPR interviews \\ facilitated critical feedback but also caused some distress. Interviews gave more \\ information about organisational level contextual factors. IPR increased \\ understanding of how the intervention was being delivered and experienced. IPR \\ should be used alongside other forms of qualitative data collection. The potential \\ of IPR to impact on intervention delivery should be taken into account. Further \\ research is needed to establish for whom IPR is likely to be most effective.
}

Keywords: interpersonal process recall; collaborative care; recovery; psychosis; qualitative research methods

\section{Introduction}

Collaborative care has been identified as a potentially effective model for addressing the poorer health and social outcomes experienced by people diagnosed with schizophrenia, bipolar and other psychoses (Baker et al., 2019). The use of psychosocial interventions to support patients to become active in managing their own condition and care is a core component of collaborative care for mental health (Reilly et al., 2013). The concept of personal recovery is now widely endorsed as a basis for mental health service provision 
and so, in the development of the PARTNERS model of collaborative care for people diagnosed with psychosis, recovery was adopted as a practice orientation. Practices, such as coaching (Bora, Leaning, Moores \& Roberts, 2010), that support service users becoming empowered, are key components of recovery-focused services (Leamy et al, 2011). Coaching was therefore adopted as the specific psychosocial intervention in the model.

During the feasibility testing phase of the PARTNERS model, a qualitative formative evaluation was carried out. The aims were to establish whether the intervention was delivered and experienced as intended and identify barriers and facilitators to delivery. Reliably assessing fidelity and accessing a range of service user experiences present challenges in evaluating the delivery of recovery-focused interventions for people diagnosed with psychosis. Previous similar studies have identified that the use of semi-structured interviews and focus groups may be limited by recall bias (Leamy et al., 2014; Wallace et al., 2016). Furthermore, practitioner accounts in interviews may be influenced by their attitude towards the intervention; practitioner perceptions that they are already working in a recovery-oriented way have been identified as a common barrier to implementation of recovery-focused interventions (Whitley et al., 2009; Leamy et al., 2014). Practitioners may therefore report that they are delivering recovery-focused care when their practice is not consistent with the model. Additionally, service user accounts may be influenced by concerns about negatively evaluating their workers or reflect internalised stigma related to mental health diagnosis (Tew, 2005). This may be particularly relevant to service users diagnosed with psychosis (Huggett et al., 2018)

In order to increase rigour, two other evaluations of recovery-focused 
programmes have triangulated data from interviews with direct researcher observations (Whitley et al., 2009; Whitley \& Siantz, 2012). However, these provided limited insight into any attitudinal barriers to implementation, as the reasoning process leading to staff actions were not examined. They may also not have accurately captured how service users experienced what had been delivered. A potential method for addressing such limitations is Interpersonal Process Recall (Elliot \& Shapiro, 1988).

\section{Interpersonal process recall}

Interpersonal process recall (IPR) involves audio or video recording of interactions between practitioners and service users and playing it back to them shortly after the session, pausing at significant moments chosen by the participant, and interviewing them about their experience. IPR yields three qualitative data sources: the consultation, the practitioner interview and the service user interview. IPR is thought to allow access to practitioner decision-making processes and immediate impacts on service users and overcome issues relating to delayed recall (Larsen, Fleseker \& Stege, 2008).

IPR has been found to stimulate more accurate recall than other methods (Mcleod, 2001). It has also been used to build a detailed understanding of therapists' internal processes (Rober, Elliott, Buysse, Loots \& De Coorte, 2008). Of particular relevance to this study relating to a novel intervention, it has been used to identify factors affecting the application of newly acquired knowledge and skills by trainee therapists, (Burges, Rhodes \& Wilson, 2011; Cartwright \& Gardner, 2016). IPR has also been used effectively to explore service users' perspectives of therapy processes, including sensitive areas such as experiences of sadness and disengagement, possibly as it allows participants to take a detached, reflective stance (Frankel \& Levitt, 2009; Henretty, Levitt \& Matthews, 2008). Particularly related to the aims of the formative 
evaluation, IPR has been used to gain insight into whether a particular approach to therapy has worked as intended (Jones, Latchford \& Tober, 2016) and explore discordance between practitioner and service user experiences of the same interaction (Larsen et al. 2008; Mcleod 1990). Additionally, consistent with the principles of the PARTNERS model, IPR has been identified as empowering for participants, enabling them to contribute more actively to the interpretation of their responses (Larsen et al., 2008, Macaskie, Lees \& Freshwater, 2015).

IPR presents a number of challenges, including the logistical requirements of scheduling and access to equipment. It is also time consuming, with one 50 minute therapy session resulting in 3 hours of interview with each participant (Elliott \& Shapiro, 1988; Larsen et al., 2008). Consequently, a short form, Brief Structured Recall was developed, in which only events identified in advance by the service user as significant were replayed. Brief Structured Recall has been found to be as effective as full IPR (Elliott \& Shapiro, 1988) and has been used successfully to study interactions between patients with mental health problems and GPs (Cape et al., 2010).

Other challenges of IPR include that it may be emotionally exposing for both service users and practitioners, in sharing a deeply personal process with an observer (Larsen et al., 2008). Additionally, some participants may find it difficult to make the conceptual shift from focussing on the content to the process of the interaction. Larsen et al. (2008) offer several strategies to mitigate these difficulties but suggest that IPR may work best with psychologically minded participants and be less successful with people experiencing psychosis, who may not be able to tolerate observing themselves.

The Brief Structured Recall form of IPR was adopted in the formative evaluation of the PARTNERS model, in combination with semi-structured interviews. It 
was thought that this would increase knowledge about practitioner understanding and service user experiences of the psychosocial component of the intervention, while ensuring that data collection and interpretation were manageable within the timescales of the pilot study. This separate, methodological study was conducted in order to assess whether the addition of IPR achieved the aim of increasing knowledge about the interpersonal intervention's delivery and impact. Additionally, this study aimed to explore the impact of the IPR process on participants, to inform decision making about its suitability for future similar studies.

\section{Method}

The formative evaluation consisted of the small-scale delivery of the PARTNERS intervention in three English regions, with qualitative data collected from all stakeholders. Ethical approval was obtained from the relevant NHS Research Ethics Committee.

The data presented here were drawn from one site, as it was not possible to schedule sufficient IPR interviews with the practitioner delivering the intervention in the other two sites. Consequently multiple sets of associated semi-structured interview and IPR data were not available for these sites.

In the site from which sufficient data was obtained, the intervention was delivered in two GP practices in a rural area. The practitioner delivering the intervention, known as a care partner, was a qualified social worker working with the local secondary care Community Mental Health Team (CMHT). The intervention was delivered over a period of ten months to eight service users. Potential participants were identified through screening of CMHT and GP records. Inclusion criteria were: a diagnosis of schizophrenia or bipolar, mild symptoms in the last year, not currently 
receiving acute intervention. Although the intervention was novel some participants had previously worked with the care partner in his secondary care role.

\section{Participants}

All service users receiving the intervention and the care partner were invited to participate in the formative evaluation and all gave written informed consent. Data was collected from seven service users and the care partner. Data from only four service users and the care partner were included in the analysis for this study (see 'Data analysis' section).

\section{Procedure}

Topic guides for the semi-structured interviews were developed by the research team in collaboration with the PARTNERS Lived Experience Advisory Panels. The topic guides explored the delivery and impact of the PARTNERS intervention, as described in the manual and associated service user guide. Copies of the topic guides are available on request from the authors.

The procedure for IPR was adapted from the original Brief Structured Recall process, in order to focus on the aims of the formative evaluation rather than issues identified as significant by service users. Intervention sessions were audio recorded and the recordings were reviewed by multiple researchers, who identified five or six short excerpts where the intervention appeared not to have been delivered or experienced as intended. The chosen excerpts were discussed to ensure that those believed to be most likely to stimulate discussion of barriers to delivery or acceptability were selected. The care partner and service user were then interviewed separately in relation to the same excerpts. The excerpts were replayed and the participants were asked for their 
views and prompted to discuss what was and was not helpful and what could have been changed.

Researchers contacted consenting participants by phone to arrange interviews and session recordings. One service user was not available during the relevant time period and another was not available for their semi-structured interview but did participate in a session recording and IPR interview. One service user did not consent to having a session recorded and another was not approached, on the advice of the care partner that it would be detrimental to the service user's mental health. While researchers were committed to empowering service users to make informed decisions about participation in the research process they were also committed to the wellbeing of participants and believed it was important to be guided by a clinician.

Interviews were conducted by four researchers, one of whom was a service user researcher. Interviewers received training in qualitative methods and interviewing techniques relevant to their level of experience from other members of the research team. Training was provided in telephone and face-to-face sessions, with individual feedback provided on early interviews. A team member with experience of conducting IPR interviews demonstrated the process and provided guidance to other interviewers.

Semi-structured interviews lasted between one and two hours and IPR interviews lasted about one hour. Most interviews took place at the participating GP practice, with one taking place at the service user's home; all participants were alone with the interviewer. All participants had previously met their interviewer during recruitment to the pilot study. The service user researcher disclosed her experience of using services to participants; the Participant Information Sheet specified that other interviewers were 'very experienced mental health researchers'. All interviews were 
audio recorded and interviewers kept field notes using an agreed template. Service users received a $£ 10$ shopping voucher for each data source.

\section{Data analysis}

All data sources were transcribed and imported into NVivo 11. The analysis for this study was conducted by two researchers. Familiarisation with the data took place during the analysis for the wider formative evaluation. During this process both researchers noted differences in the impression formed of the interactions between the care partner and service user from different data sources and the ways in which the IPR process had impacted on the participants. It was decided to make a systematic comparison between the themes present in semi-structured interviews, session recordings and IPR, and explore the advantages and disadvantages of using IPR. It was decided to only include data from service users for whom there was both a semi-structured interview and an IPR interview available: a total of seventeen data sources from four service users and the care partner. The data sources available and those included in the analysis for this study are shown in Figure 1.

\section{[FIGURE 1 ABOUT HERE]}

In order to explore differences in the content of the data sources, a framework analysis (Ritchie \& Spencer, 1994) was used. An analytical framework was developed, refined and applied to all the transcripts included in the analysis by the two researchers, with regular meetings to check for consistency and discuss interpretations. It was identified that 'empowerment' was the most frequently applied category, and was often doublecoded with other elements of the model. It was therefore decided to focus the analysis on the 'empowerment' category, as it was likely to be representative of the care 
partner's overall approach. A thematic chart was devised with data from each source grouped together and a summary of the data coded under each heading was transposed into the chart. In order to enable a more systematic comparison, further themes and subthemes were then identified and defined within the transposed data and a table was constructed to show which themes were present or absent in each data source.

\section{Results}

Demographics of the participants are shown in Table 1. Of the service users included in this study, one had previously worked with the care partner.

\section{[TABLE 1 ABOUT HERE]}

The themes and sub-themes identified through the final stage of the analysis are summarised in Table 2. The themes relating to the presence or absence of empowerment processes were labelled as 'done with' and 'done to', derived from the care partner's use of these terms to characterise a fundamental distinction between the PARTNERS intervention and traditional community mental health practice in his semi-structured interview.

\section{[TABLE 2 ABOUT HERE]}

As the focus of this study is a comparison between the different data collection methodologies, the content of the themes will not be described in detail; alternatively differences between the sources in which they occurred will be highlighted. The data 
sources in which each theme was present are shown in Table 3, which illustrates that some themes were only present in certain sources.

\section{[TABLE 3 ABOUT HERE]}

\section{Themes present in semi-structured interviews}

Of the two 'empowerment processes' themes, only 'done with' was present in the semistructured interviews with either care partners or service users. There were many examples of the sub-themes reflecting empowering care partner contributions and service users being empowered to take small steps towards their goals and growing in confidence. There also appeared to be an interactive effect whereby service users' progress reinforced the care partner's confidence in using empowering practices, further strengthening their self-belief and resolve to take positive steps, creating an interpersonal virtuous 'cycle of success':

he said it was great because I was one of three really good things and they so rarely happen in his job And so that... reinforces my confidence and makes me feel good about myself... So... it's almost a two-way, you know, if I can walk out of here feeling that I've made [care partner] feel a bit better, that makes me feel even better about the way [care partner]'s made me feel [SU4- semi-structured interview]

What I hadn't pictured is how much I enjoy coming from those sessions... I'm usually quite 'Wow', you know, it had a real impact on, you know, the 
collaborative work that we've done, I'm seeing some really quick results here [care partner- semi-structured interview]

This suggested that the care partner was delivering the intervention and that empowering practice was resulting in service user empowerment. However, this understanding was modified as a result of the data from the additional sources; this will be discussed further below.

In relation to the 'contextual factors', semi-structured interviews provided the richest source of information about organisational barriers and facilitators: the servicelevel policies and priorities that supported, or not, the delivery of empowerment. Subthemes that were present only in the semi-structured interviews were the availability of supervision and the focus of the PARTNERS intervention on individual priorities and improving wellbeing rather than service priorities and crisis management.

\section{Interviewer: Is that the main thing that you think is different to the support you've had before?}

SU4: Yeah because there is no goal orientation ... in the conventional psychiatric system... they don't seem to believe that apart from some level of symptom relief... there is any hope of getting people back in to, or maybe this deluded belief that if you get people on the right medication they will magically sort themselves out and get their own lives back together [SU4semi-structured interview]

He's on Clozapine so, you know, he'll be reviewed by myself and the, er, psychiatrist, and that would be pretty much the only amount of input we 
could give [SU1] unless his presentation changes to sort of pre-crisis, ...it's... having a set bit of time away with somebody who has not been a priority on my caseload... I've learned so much about [SU1] and his life in PARTNERS ... that I would have never have probably known in secondary. [care partner- semi-structured interview]

\section{Themes present in the intervention sessions}

The 'done with' theme was also present in all the intervention sessions. However, the 'done to' theme was also identified in many of the intervention sessions, with subthemes reflecting ways in which researchers interpreted the care partner as prioritising his own construction of the service users' experience. Service users appeared to typically respond by acquiescing. In the sequence below, the care partner responds to the service user stating that he has been "getting messages off the telly" by making a distinction between "psychotic" experience and thoughts "we can all have", rather than exploring the service user's experience and preferred narrative. The service user then changes the subject and accepts the care partner refocusing the session on tasks that the care partner needs to complete.

CP: When you say messages from the TV, you mean messages as in something you're watching not something you're hearing or seeing.

SU1: No, something I'm watching.

CP: Yeah, okay.

SU1: Yeah.

\section{CP: But not psychotic kind of?}


SU1: I don't know, people saying, you know, it's a difference between having thoughts and hearing voices, I can't, people, some people ask do you hear voices? But I say I have thoughts, whether they're voices, I don't know?

CP: Yeah, I guess, I guess the difference is, we, we all can have thoughts and can sort of make audible thoughts

SU1: Well, yeah, you have to make decisions don't you?

CP: In our minds, but I guess the difference is when those thoughts are there [inaudible]

SU1: [talking over care partner] See I'll go down to [place], check out how much money I've got and make a decision based on that, you know, because if I overspend, I overspend more money and then I get short at the end of the two weeks, but this voucher is ideal.

\section{CP: OK [rustling papers]}

SU1: I wasn't expecting that today

CP: Nice, I didn't know that they were giving vouchers out, it's really good

SU1: Yeah, I go in to [name of shop] often so you know

CP: Um, so I've got this individual goal record

SU1: OK

CP: Yeah, I'm just going to write down on here

SU1: Yeah? Yeah right

The presence of 'done to' processes in intervention sessions suggested that the care partner had not consistently delivered an empowering approach. The intervention 
session data did not allow the identification of potentially contributing contextual factors, or indicate how service users might have experienced a 'done to' approach. These themes were however identified in the IPR interviews.

\section{Themes present in the care partner IPR interviews}

The care partner IPR interviews were the only data source in which the sub-theme of 'care partner barriers' was identified. This increased understanding of potential influences on the care partner's approach. It appeared that as well as the knowledge and skills provided by the PARTNERS intervention, the care partner drew on attitudes and knowledge developed through working in a secondary care culture that gave primacy to narratives concerned with managing illness and risk. The care partner appeared most likely to draw on such narratives when he was experiencing increased anxiety about service users and, in one case, as a result of having worked with the service user previously in a CMHT context. In reflecting on the excerpt above, the care partner constructed the service user's experiences as symptoms and possible signs of increasing risk:

I would be thinking that that's a little bit concerning if he's starting to have messages and these kind of early indicators. I perhaps would want to explore that a bit more... But also knowing that [SU1] ... has a ... treatment resistant schizophrenia, that those kind of symptoms are quite common. [care partner -IPR interview re SU1] 
This excerpt also illustrates that the care partner used the IPR to reflect on his practice and identify how it could be improved. This 'care partner self-reflection and learning' was present in all of the care partner's IPR interviews and, in many instances, the care partner identified that his practice had reflected a 'done to' approach:

I think from that... perhaps my agenda and perhaps not his...we could have identified a further goal...but I guess that needs to come from [SU4] and not me. [care partner-IPR interview re SU4]

\section{Themes present in the service user IPR interviews}

The 'done to' theme was also present in the IPR interviews of three out of the four service users.

I think I've used the term 'invalidation' several times already, um, and that was something where I felt that [care partner] was invalidating what I was saying, rather than really addressing it [SU4- IPR interview]

This contrasts with the absence of this theme from the service user semi-structured interviews.

The 'service user response' theme indicated that some participants experienced distress. This was either the result of focussing on difficulties, or for one service user, hearing his voice on the recording. Both interfered with participants' ability to reflect on the care partner's actions:

SU1: [laughs] Oh dear, oh I do sound so common. 


\section{Interviewer: OK, so was there anything helpful about that discussion, would you say? \\ SU1: Um, oh, I, um, I hate it when I talk like that in some ways, 'cos people pick up on it and think you must be mentally ill because you're saying this.}

This issue was most significant for SU1 and it may be relevant that 'service user barriers' to empowerment were also most likely to be identified in relation to SU1. When asked about his view of the care partner's response to his concerns about hearing voices in the extract above, his reply indicated that he had internalised a potentially stigmatising medicalised narrative:

\section{Interviewer: I didn't know if you were asking [care partner] a question, and I didn't know if you wanted more information or you - \\ SU1: Well, I think it was a rhetorical question really. But, yeah, the only answer is you must be mentally ill}

This suggested that the effectiveness of IPR in supporting critical reflection for service users could potentially be mitigated by the internal barriers to empowerment that were identified. 


\section{Discussion}

Through the systematic comparison of data sources used in the formative evaluation of the PARTNERS intervention, it was identified that different themes were present in semi-structured interviews and the additional data sources created through the IPR process.

The themes present in the intervention sessions and the IPR interviews indicated that, while there was evidence of fidelity, the intervention was not being delivered as consistently as it appeared from the semi-structured interviews. While intervention sessions revealed inconsistencies in the delivery of the intervention, they provided limited information about the factors contributing to, and the impact of, these inconsistencies. The IPR interviews enabled a greater understanding of care partner internal barriers and service user responses. However, some themes relating to organisational level contextual factors were only present in the semi-structured interviews.

Similar to previous studies, these findings indicate that IPR can be used to understand therapist internal processes (Rober et al., 2008), specifically barriers to the application of new interventions (Burges et al, 2011; Cartwright \& Gardner, 2016). In this case, IPR supported the practitioner to recognise when his practice diverged from the intervention. These findings are also consistent with previous studies that have found that IPR can enable service users to articulate the impact of particular therapist behaviours and approaches (Frankel \& Levitt, 2009; Jones et al., 2016). IPR appeared to enable some service users to reflect more critically on their experiences and in that sense was empowering, as has previously been suggested (Larsen et al., 2008). Incorporating IPR as a methodology in evaluating the delivery of novel, recovery focussed interventions could therefore potentially overcome the limitations of using 
semi-structured interviews alone. However, these findings also indicate that semistructured interviews were more effective in capturing contextual information, possibly as they created an opportunity for participants to reflect generally on the key characteristics of the intervention and contrast this to their experience of standard care. The impact of the IPR process on participants was also explored. Most of the service users engaged successfully with the IPR process. However, where an impact of the IPR process on service users was identified, it was related to distress. As suggested by Larsen et al., (2008) for one service user this appeared to be related to the experience of observing himself. Additionally, this service user was identified as experiencing internal barriers to empowerment, which appeared to impact on his ability to offer views that might be inconsistent with professional opinion. This suggests that the use of IPR may not enable all service users to overcome the effects of internalised stigma.

The impact of the IPR process on the care partner was increased self-awareness and understanding of how his practice could be more consistent with the intervention. Similar effects have been found in other IPR studies (Cartwright \& Gardener, 2016; Larsen et al. 2008). IPR was developed as a therapy training and supervision technique, and is a well-established supervision methodology (Walker, 2010). It is thought to be most effective for supporting critical reflection on subtle interpersonal dynamics (Griffith \& Frieden, 2000; Meekums, Macaskie \& Kapur, 2016). This suggests that IPR has the potential to support practitioners in reflecting on, and developing, their practice in relation to empowerment. The use of IPR for data collection could therefore enhance implementation in relation to recovery-focussed interventions. While this could be beneficial in terms of increasing fidelity, researchers would need to be aware of the potential for Hawthorne effects when using this approach. 
This study is limited by a small dataset, representing the experiences of one practitioner and four service users. Furthermore the sample was largely self-selecting and it is possible that only those participants with the greatest capacity for reflecting in the way demanded by IPR took part, making the technique appear more effective. The lack of a complete data set in two of our sites suggests that the increased complexity of arranging IPR interviews, and potentially the reluctance of participants to engage in a psychologically exposing process, may be a barrier to effective data collection though IPR.

Our sample was exclusively white British; this reflected the demographic composition of the site. However, psychosis is known to be identified most frequently in people from ethnic minority backgrounds (King et al., 2005). More research is therefore needed to establish whether IPR is an effective technique with more diverse groups.

\section{Conclusion and recommendations}

The inclusion of IPR in the formative evaluation of a recovery-focused intervention for people with psychosis increased understanding of how the intervention was delivered and experienced and the factors that influenced delivery. However, additional information about contextual factors was solely identified through the use of semi-structured interviews. This indicates that IPR should be considered as a methodology for evaluating such interventions but should be used alongside other methodologies in order to maximise understanding of facilitators and barriers to implementation of an intervention.

While IPR supported the majority of the service user participants to reflect critically on the intervention, it was less successful in one case and caused some 
distress. Although possible contributing factors were identified, more research is needed to understand for whom IPR is most likely to be effective, and the role of factors such as psychosis, ethnic background and internalised stigma. There is also a need to develop and evaluate alternative procedures which could similarly overcome recall biases and facilitate critical reflection without causing distress. Possibilities include the use of transcripts instead of playing back recordings and interview schedules focusing on specific recent interactions rather than general experiences of the intervention.

In addition to increasing the understanding of researchers, the use of IPR increased the care-partner's understanding of how he was delivering the intervention. IPR thus also has a potential role in supporting the implementation of recovery-focussed interventions for people with psychosis. The effect of participating in IPR on practitioner behaviour should be taken into consideration if data is being collected longitudinally.

\section{Disclosure of Interest}

The authors report no conflict of interest 


\section{References}

Baker, E., Gwernan-Jones, R, Britten, N. Cox, M., McCabe, C., Retzer, A., Gill, L., Plappert, H., Reilly, S., Pinfold, V., Gask, L., Byng, R. Birchwood, M. (2019). Refining a model of collaborative care for people with a diagnosis of bipolar, schizophrenia or other psychoses in England: a qualitative formative evaluation. BMC Psychiatry, 19,7.

Bora, R., Leaning, S., Moores, A. \& Roberts, G. (2010). Life coaching for mental health recovery: the emerging practice of recovery coaching. Advances in Psychiatric Treatment, 16, 459-467.

Burges, S., Rhodes, P. \& Wilson, V. (2013). Exploring the in-session reflective capacity of clinical psychology trainees: An interpersonal process recall study. Clinical Psychologist, 17, 122-130.

Cape, J., Geyer, C., Barker, C., Pistrang, N., Buszewicz, M., Dowrick, C. \& Salmon, P. (2010). Facilitating understanding of mental health problems in GP consultations: A qualitative study using taped-assisted recall. British Journal of General Practice, 60 (580), 837-845.

Cartwright, D. \& Gardner, M. (2016). Trainee difficulties with therapeutic engagement: A qualitative study of in-session critical incidents. South African Journal of Psychology, 46(2), 254-265.

Elliott. R. \& Shapiro, D. A. (1988). Brief Structured Recall: A more efficient method for studying significant therapy events. British Journal of Medical Psychology, $61,141-153$.

Frankel, Z. \& Levitt, H. M. (2009). Clients' experiences of disengaged moments in psychotherapy: A grounded theory analysis. Journal of Contemporary Psychotherapy, 39, 171-186. 
Griffith, B.A. \& Frieden, G. (2000). Facilitating reflective thinking in counsellor education. Counselor Education and Supervision, 40(2), 82-93.

Henretty, J. R., Levitt, H. M. \& Matthews, S.S. (2008). Clients' experiences of moments of sadness in psychotherapy: A grounded theory analysis. Psychotherapy Research, 18(3), 243-255.

Huggett, C., Birtel, M. D., Awenat, Y. F., Fleming, P., Wilkes, S., Williams, S. \& Haddock, G. (2018). A qualitative study: Experiences of stigma by people with mental health problems. Psychology and Psychotherapy: Theory, Research and Practice, 91, 380-397.

Jones, S. A., Latchford, G. \& Tober, G. (2016). Client experiences of motivational interviewing: An interpersonal process recall study. Psychology and Psychotherapy: Theory, Research and Practice, 89 (1), 97-114.

King, M., Nazroo, J., Weich, S., McKenzie, K., Bhui, K., Karlsen, S., Stansfeld, S., Tyrer, P., Blanchard, M., Lloyd, K., McManus, S., Sproston, K. \& Erens, B. (2005) Psychotic symptoms in the general population of England: A comparison of ethnic groups (The EMPIRIC study). Social Psychiatry and Psychiatric Epidemiology, 40, 375-81.

Larsen, D., Fleseker, K. \& Stege, R. (2008). Qualitative interviewing using interpersonal process recall: Investigating internal experiences during professional-client conversations. International Journal of Qualitative Methods, 7(1), 18-37.

Leamy, M., Bird, V., Le Boutillier, C., Williams, J. \& Slade, M. (2011). A conceptual framework for personal recovery in mental health: Systematic review and narrative synthesis. British Journal of Psychiatry, 199, 445-452. 
Leamy, M., Clarke, E., Le Boutillier, C., Bird, V., Janosik, M., Sabas, K., Riley, G., Williams, J. \& Slade, M. (2014) Implementing a complex intervention to support personal recovery: A qualitative study nested within a cluster randomised controlled trial, PLoS ONE, 9, e97091.

Macaskie, J., Lees, J \& Freshwater, D. (2015). Talking about talking: Interpersonal process recall as an intersubjective approach to research. Psychodynamic Practice, 21(3), 226-240.

McLeod, J. (1990). The client's experience of counselling and psychotherapy. In D. Mearns \& W. Dryden (Eds.) Experiences of counselling in action (pp 1-19). London: Sage.

Mcleod, J. (2001) Qualitative Research in Counselling and Psychotherapy. London: Sage.

Meekums, B., Macaskie, J. \& Kapur, T. (2016). Developing skills in counselling and psychotherapy: A scoping review of Interpersonal Process Recall and Reflecting Team methods in initial therapist training. British Journal of Guidance and Counselling, 44 (5), 504-515.

Reilly, S., Planner, C., Gask, L., Hann, M., Knowles, S., Druss, B., Lester, H. (2013) Collaborative care approaches for people with severe mental illness. Cochrane Database of Systematic Reviews, 11.

Ritchie, J. \& Spencer, L. (1994). Qualitative data analysis for applied policy research. In A. Bryman \& R. G. Burgess (Eds.) Analysing Qualitative Data (pp173-194). Abingdon: Taylor and Francis.

Rober, P., Elliott, R., Buysse, A., Loots, G. \& De Corte, K. (2008). Positioning in the therapist's inner conversation: A dialogical model based on a grounded theory 
analysis of therapist reflections. Journal of Marital and Family Therapy, 34, 406421.

Tew, J. (2005) Power relations, social order and mental distress. In J. Tew (Ed.) Social Perspectives in Mental Health (pp71-89). London: Jessica Kingsley.

Walker, J. A. (2010). Supervision techniques. In N. Ladany and L. J. Bradley (Eds.) Counselor Supervision $\left(4^{\text {th }}\right.$ ed., pp. 97-122). New York, NY: Routledge. Wallace, G., Bird, V., Leamy, M., Bacon, F., Le Boutillier, C., Janosik, M., Macpherson, R., Williams, J. \& Slade, M. (2016). Service user experiences of REFOCUS: A process evaluation of a pro-recovery complex intervention, Social Psychiatry and Psychiatric Epidemiology, 51, 1275-1284.

Whitley, R., Gingerich, S., Lutz, W. J. \& Mueser, K. T. (2009). Implementing the Illness Management and Recovery program in community mental health settings: Facilitators and barriers. Psychiatric Services 60(2), 202-209.

Whitley, R. \& Siantz, E. (2012). Recovery centers for people with a mental illness: An emerging best practice? Psychiatric Services, 63(1), 10-12. 
Figure 1. Data sources included and not included in the analysis

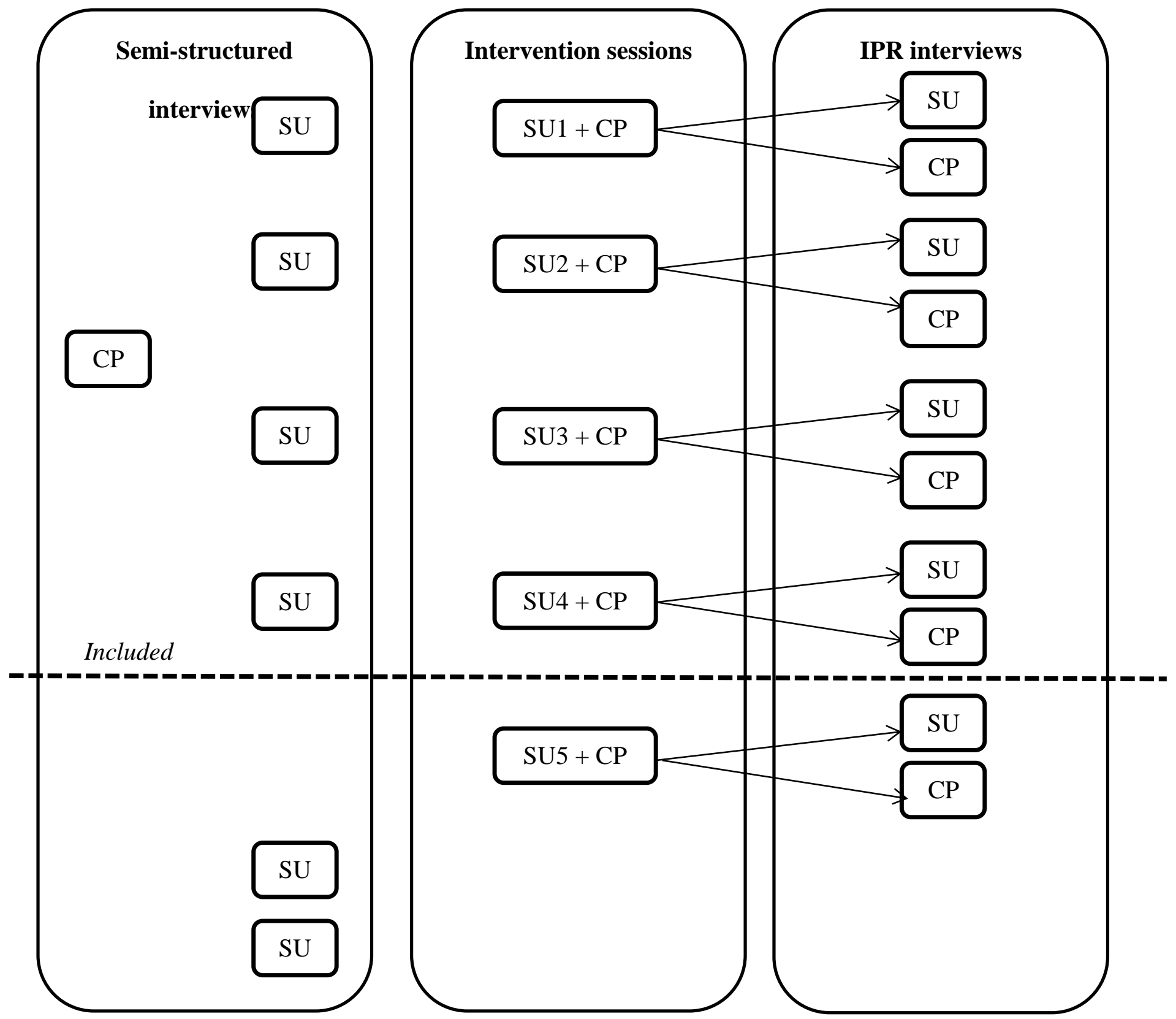

Data sources included in the analysis are shown above the dotted line. 
Table 1. Demographic data

\begin{tabular}{lll}
\hline & Service user & Practitioner \\
\hline Gender $(\mathrm{N}, \%)$ & & \\
\hline $\begin{array}{l}\text { Female } \\
\text { Male }\end{array}$ & $1(25)$ & $1(100)$ \\
& $3(75)$ & - \\
\hline Age (mean, range) & $45.8(31-52)$ & - \\
\hline Ethnicity (N, \%) & & $1(100)$ \\
\hline White British & $4(100)$ & \\
\hline Diagnosis (N, \%) & & \\
\hline $\begin{array}{l}\text { Schizophrenia } \\
\text { Bipolar }\end{array}$ & $1(25 \%)$ & \\
\hline
\end{tabular}


Table 2. All themes identified in the data

Theme 1. Empowerment processes

\section{1 'Done with'}

\subsubsection{Care partner}

contribution

- acts as 'real' person

- sees service user as individual, beyond diagnosis

- offers choice and control in care

- engages in positive risk taking

- supports control over mental health

\subsubsection{Service user response}

- Takes small steps towards agreed goals

- Growing confidence

\subsubsection{Interactive effect}

- cycle of success

\section{2 'Done to'}

1.2.1 Care partner

contribution

- Unresponsive to SU concerns or interests

- Makes dissonant interpretation, imposes meaning or pathologises experience

- Prioritises risk management

\subsubsection{Service user response}

- Acquiesces

- Challenges

\subsubsection{Interactive effect}

- care partner feels need to take control

- care partner reverts to 'done with'

\section{Theme 2. Contextual influences}

\subsection{Care partner characteristics}

\subsubsection{Care partner facilitators}

- personal style consistent with intervention ethos

\subsection{Service user characteristics}

\subsubsection{Service user facilitators}

- motivated to change

- stability of mental health

\subsection{Organisational} characteristics

2.3.1 Organisational facilitators

- Sufficient time

- Supervision provided

- Prioritises individualised approach

- Prioritises improving wellbeing

\subsubsection{Service user barriers}

- internalised negative view

- increasing distress

\subsubsection{Organisational barriers}

- Time pressured

- Absence of supervision

- Standardised approach to care

- Prioritises crisis management service user from CMHT role

- anxiety and protectiveness 
Table 3. Themes identified in each data source

\begin{tabular}{|c|c|c|c|c|c|c|c|c|c|c|c|c|c|c|c|c|c|}
\hline & \multirow{2}{*}{$\begin{array}{l}\text { Care } \\
\text { partner } \\
\text { interview } \\
\text { CP1 } \\
\end{array}$} & \multicolumn{4}{|c|}{ Service user interview } & \multicolumn{4}{|c|}{ Intervention session } & \multicolumn{4}{|c|}{$\begin{array}{l}\text { Care partner IPR } \\
\text { interview }\end{array}$} & \multicolumn{4}{|c|}{$\begin{array}{l}\text { Service user IPR } \\
\text { interview }\end{array}$} \\
\hline & & SU1 & SU2 & SU3 & SU4 & SU1 & SU2 & SU3 & SU4 & SU1 & SU2 & SU3 & SU4 & SU1 & SU2 & SU3 & SU4 \\
\hline 'Done with' & $\mathrm{X}$ & $\mathrm{X}$ & $\mathrm{X}$ & $\mathrm{X}$ & $\mathrm{x}$ & $\mathrm{x}$ & $\mathrm{x}$ & $\mathrm{x}$ & $\mathrm{x}$ & $\mathrm{X}$ & $\mathrm{X}$ & $\mathrm{X}$ & $\mathrm{X}$ & $\mathrm{x}$ & $\mathrm{X}$ & $\mathrm{X}$ & $\mathrm{X}$ \\
\hline 'Done to' & & & & & & $\mathrm{x}$ & $\mathrm{X}$ & & $\mathrm{x}$ & $\mathrm{x}$ & $\mathrm{X}$ & $\mathrm{X}$ & $\mathrm{X}$ & $\mathrm{x}$ & $\mathrm{x}$ & & $\mathrm{X}$ \\
\hline $\begin{array}{l}\text { Care partner } \\
\text { barriers }\end{array}$ & & & & & & & & & & $\mathrm{x}$ & & $\mathrm{x}$ & $\mathrm{x}$ & & & & \\
\hline $\begin{array}{l}\text { Care partner } \\
\text { facilitators }\end{array}$ & $\mathrm{x}$ & & & & & & & & & $\mathrm{x}$ & & & & & & & \\
\hline $\begin{array}{l}\text { Service user } \\
\text { barriers }\end{array}$ & $\mathrm{x}$ & & & & & $\mathrm{x}$ & & & & $\mathrm{x}$ & $\mathrm{X}$ & & & $\mathrm{x}$ & & & \\
\hline $\begin{array}{l}\text { Service user } \\
\text { facilitators }\end{array}$ & $\mathrm{x}$ & & $\mathrm{X}$ & $\mathrm{X}$ & $\mathrm{X}$ & & & & $\mathrm{x}$ & & $\mathrm{X}$ & $\mathrm{X}$ & & $\mathrm{x}$ & & & $\mathrm{X}$ \\
\hline $\begin{array}{l}\text { Organisational } \\
\text { barriers }\end{array}$ & $\mathrm{x}$ & & & & & & & & & $\mathrm{x}$ & $\mathrm{X}$ & & & & & & \\
\hline $\begin{array}{l}\text { Organisational } \\
\text { facilitators }\end{array}$ & $\mathrm{x}$ & & & $\mathrm{x}$ & $\mathrm{X}$ & & & & & $\mathrm{x}$ & & & & & & & \\
\hline Impact of IPR & & & & & & & & & & $\mathrm{X}$ & $\mathrm{X}$ & $\mathrm{X}$ & $\mathrm{X}$ & $\mathrm{X}$ & & & $\mathrm{X}$ \\
\hline
\end{tabular}

